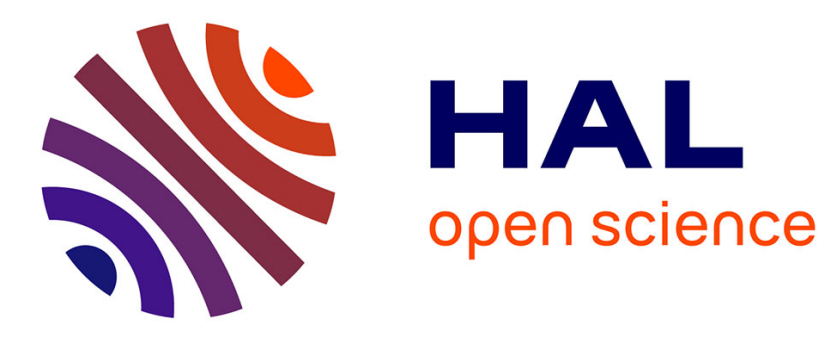

\title{
On Combinatorial Proofs for Logics of Relevance and Entailment
}

\author{
Matteo Acclavio, Lutz Strassburger
}

\section{To cite this version:}

Matteo Acclavio, Lutz Strassburger. On Combinatorial Proofs for Logics of Relevance and Entailment. WoLLIC 2019 - 26th International Workshop on Logic, Language, Information, and Computation, Jul 2019, Utrecht, Netherlands. pp.1-16, 10.1007/978-3-662-59533-6_1 . hal-02390426

\section{HAL Id: hal-02390426 \\ https://hal.inria.fr/hal-02390426}

Submitted on 3 Dec 2019

HAL is a multi-disciplinary open access archive for the deposit and dissemination of scientific research documents, whether they are published or not. The documents may come from teaching and research institutions in France or abroad, or from public or private research centers.
L'archive ouverte pluridisciplinaire HAL, est destinée au dépôt et à la diffusion de documents scientifiques de niveau recherche, publiés ou non, émanant des établissements d'enseignement et de recherche français ou étrangers, des laboratoires publics ou privés. 


\title{
On Combinatorial Proofs for Logics of Relevance and Entailment
}

\author{
Matteo Acclavio ${ }^{1}$ and Lutz Straßburger ${ }^{2}$ \\ ${ }^{1}$ Università Roma Tre http://matteoacclavio.com/Math.html \\ ${ }^{2}$ Inria Saclay http://www.lix.polytechnique.fr/Labo/Lutz.Strassburger/
}

\begin{abstract}
Hughes' combinatorial proofs give canonical representations for classical logic proofs. In this paper we characterize classical combinatorial proofs which also represent valid proofs for relevant logic with and without the mingle axiom. Moreover, we extend our syntax in order to represent combinatorial proofs for the more restrictive framework of entailment logic.
\end{abstract}

Keywords: Combinatorial Proofs - Relevant Logic · Entailment Logic - Skew fibrations · Proof Theory

\section{Introduction}

Combinatorial proofs have been conceived by Hughes [11] as a way to write proofs for classical propositional logic without syntax. Informally speaking, a combinatorial proof consists of two parts: first, a purely linear part, and second, a part that handles duplication and erasure. More formally, the first part is a variant of a proof net of multiplicative linear logic (MLL), and the second part is given by a skew fibration (or equivalently, a contraction-weakening map) from the cograph of the conclusion of the MLL proof net to the cograph of the conclusion of the whole proof. For the sake of a concise presentation, the MLL proof net is given as a cograph together with a perfect matching on the vertices of that graph. An important point is that in order to represent correct proofs, the proof nets have to obey a connectedness- and an acyclicity-condition.

To give an example, we show here the combinatorial proof of Pierce's law $((a \rightarrow b) \rightarrow a) \rightarrow a$, which can be written in negation normal form (NNF) as $(\bar{a} \vee b) \vee \bar{a}) \vee a:$
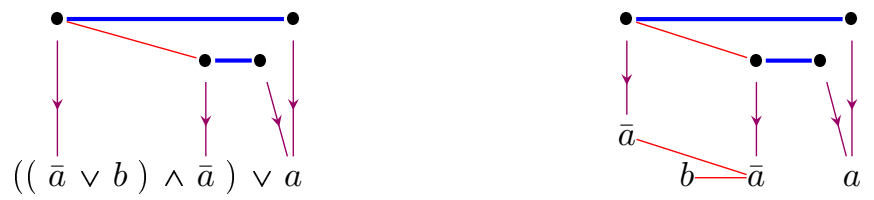

On the left above, we have written the conclusion of the proof as formula, and on the right as cograph, whose vertices are the atom occurrences of the formula, and whose edges are depicted as regular (red) lines. The linear part of the proof is given by the cograph determined by the four vertices and the regular (red) 
edge in the upper half of the diagram. The perfect matching is indicated by the bold (blue) edges. Finally, the downward arrows describe the skew fibration. In our example there is one atom in the conclusion (the $a$ ) that is the image of two vertices, indicating that it is the subject of a contraction in the proof. Then there is an atom (the $b$ ) that is not the image of any vertex above, indication that it is coming from a weakening in the proof.

Relevance logics have been studied by philosophers 23 to investigate when an implication is relevant, i.e., uses all its premises. In particular, in relevance logic, the implication $A \rightarrow(B \rightarrow A)$ is rejected because the $B$ is not used to draw the conclusion $A$. In other words, we can no longer deduce $A$ from $A \wedge B$. Put in proof theoretic terms, this corresponds to disallowing the weakening rule in a proof system. Carrying this observation to our combinatorial proofs mentioned above, this says that the skew fibration, that maps the linear part to the conclusion, must be a relevant, i.e. surjective with respect of vertices and edges of the cographs. The first contribution of this paper is to show that the converse also holds. a classical combinatorial proof is a proof of relevance logic if and only if its skew fibration is surjective.

The mingle axiom is in its original form $A \rightarrow(A \rightarrow A)$ [2, p.97]. In the implication-negation fragment of relevant logic, it can be derived from the more primitive form $A \rightarrow(B \rightarrow(\bar{B} \rightarrow A)$ ) (see also [2, p.148]), which is equivalent to $(A \wedge B) \rightarrow(A \vee B)$, which is known as mix in the linear logic community. When mix is added to MLL, the connectedness-condition has to be dropped. This leads to the second result of this paper: adding mingle to relevance logic corresponds to dropping the connectedness condition from the combinatorial proofs.

Interestingly, Hughes' original version of combinatorial proofs included mix (i.e., there was no connectedness-condition). If weakening $\perp \rightarrow A$ is present, then mix is derivable, so that the presence or absence of mix does not have an effect on provability. However, when weakening is absent, as it is the case with many substructural logics, then mix/mingle has an impact on provability, and for this reason, we present combinatorial proofs in their basic form without mix, and follow the presentation in [17, using the notion of RB-cographs due to Retoré [15].

Entailment logic is a further refinement of relevance logic, insisting not only on the relevance of premises but also on their necessity (in the sense of the modal logic S4 $)^{3}$ More precisely, the logic rejects the implication $A \rightarrow((A \rightarrow A) \rightarrow A)$. In terms of the sequent calculus, this means that the two sequents

$$
\Gamma \vdash A \rightarrow B \quad \text { and } \quad \Gamma, A \vdash B
$$

are only equivalent if all formulas in the context $\Gamma$ are of shape $C \rightarrow D$ for some $C$ and $D$. If we write $A \rightarrow B$ as $\bar{A} \vee B$, then $\vee$ is not associative, as the rejected

\footnotetext{
${ }^{3}$ We do not discuss the philosophical considerations leading to this logic. For this, the reader is referred to the Book by Anderson and Belnap 2]. We take here the logic as given and discuss its proofs.
} 
$A \rightarrow((A \rightarrow A) \rightarrow A)$ would be written as $\bar{A} \vee((A \wedge \bar{A}) \vee A)$, and the accepted $(A \rightarrow A) \rightarrow(A \rightarrow A)$ as $(A \wedge \bar{A}) \vee(\bar{A} \vee A)$. The consequence of this is that in combinatorial proofs we can no longer use simple cographs to encode formulas, as these identify formulas up to associativity and commutativity of $\wedge$ and $\vee$. We solve this problem by putting weights on the edges in the graphs. This leads us to our third contribution of this paper: combinatorial proofs for entailment logic.

Outline of the paper In this paper we study the implication-negation-fragment of relevance logic. For this, we recall in Section 2 the corresponding sequent calculi, following the presentation in [13] and [5. Then, in Section 3 we introduce another set of sequent calculi, working with formulas in NNF, and we show the equivalence of the two presentations. The NNF presentation allows us to reuse standard results from linear logic. In Sections 4 and 5 , we introduce cographs and skew fibrations, so that in section 6 we can finally define combinatorial proofs for relevance logic with and without mingle. Then, in Sections 7,8 we extend our construction to the logic of entailment.

\section{Sequent Calculus, Part I}

In this section we recall the sequent calculi for the implication-negation-fragment of relevance logic (denoted by $\mathbf{R}_{\mp}$ ), of relevance logic with mingle (denoted by $\mathbf{R} \mathbf{M}_{\mathfrak{\Im}}$ ), of entailment logic (denoted by $\mathbf{E}_{\mathfrak{\Im}}$ ), and classical propositional logic (denoted by $\mathbf{C} \mathbf{L}_{\mathfrak{\Im}}$ ).

For this, we consider the class $\mathcal{I}$ of formulas (denoted by $A, B, \ldots$ ) generated by a countable set $\mathcal{A}=\{a, b, \ldots\}$ of propositional variables and the connectives $\rightarrow$ and $(\bar{\cdot})$ by the following grammar:

$$
A, B::=a|\bar{A}| A \rightarrow B
$$

A sequent $\Gamma$ in $\mathcal{I}$ is a multiset of occurrence of formulas, written as list and separated by commas: $\Gamma=A_{1}, \ldots, A_{n}$. We denote by $\overline{\Gamma^{\rightarrow}}$ a sequent of formulas in $\mathcal{I}$ of the form $\overline{A_{1} \rightarrow B_{1}}, \ldots, \overline{A_{n} \rightarrow B_{n}}$, and we write $\bar{\Gamma}$ for the sequent obtained from $\Gamma$ by negating all its formulas, i.e., if $\Gamma=A_{1}, \ldots, A_{n}$ then $\bar{\Gamma}=\bar{A}_{1}, \ldots, \bar{A}_{n}$.

In Figure 1 we give the standard sequent systems for the logics $\mathbf{E}_{\mathfrak{7}}, \mathbf{R}_{\mathfrak{7}}, \mathbf{R M}_{\tilde{\xi}}$, and $\mathbf{C L}_{\mathfrak{\jmath}}$ as given in [13:5].

Theorem 2.1 A formula is a theorem of the logic $\mathbf{E}_{\mathfrak{F}}$ (resp. $\mathbf{R}_{\mathfrak{F}}, \mathbf{R M}_{\tilde{\Im}}, \mathbf{C L}_{\mathfrak{\xi}}$ ) iff it is derivable in the sequent calculus $\mathrm{LE}_{\tilde{\xi}}$, (resp. $\mathrm{LR}_{\tilde{\xi}}, \mathrm{LRM}_{\tilde{\xi}}, \mathrm{LK}_{\tilde{\xi}}$ ). [13]

Observe that the system $\mathrm{LE}_{\mathfrak{\ddagger}}$ in Figure 1 does contain the cut-rule, whereas the other systems are cut-free. The reason is that due to the form of the EAX, the cut cannot be eliminated.

In order to obtain cut-free systems for all four logics, we move to the negation normal form in the next section. 


\begin{tabular}{|c|c|c|}
\hline$E_{\tilde{f}}$ & $\mathrm{LE}_{\tilde{\Im}}$ & AX, EAX, $\rightarrow^{\mathrm{E}}, \neg, \mathrm{C}$, cut \\
\hline $\mathbf{R}_{\tilde{7}}$ & $\mathrm{LR}_{\tilde{f}}$ & $\mathrm{AX}, \rightarrow, \rightrightarrows, \neg, \mathrm{C}$ \\
\hline $\mathrm{M}$ & $\mathrm{LRM}_{\tilde{7}}$ & $\mathrm{AX}, \rightarrow, \rightrightarrows, \neg, \mathrm{C}, \mathrm{mAX}$ \\
\hline & & $X, \rightarrow,=$ \\
\hline
\end{tabular}

$$
\begin{array}{ccc}
\overline{A, \bar{A}} \mathrm{AX} & \overline{\bar{A}, \overline{A \rightarrow B}, B} \mathrm{EAX} & \overline{\Gamma, \bar{\Gamma}} \mathrm{mAX} \\
\frac{\Gamma, A}{\Gamma, \overline{\bar{A}}} \neg & \frac{\Gamma, A \quad \bar{B}, \Delta}{\Gamma, \overline{A \rightarrow B}, \Delta} \rightarrow & \frac{\Gamma, \bar{A}, B}{\Gamma, A \rightarrow B} \rightarrow \\
\frac{\Gamma, A, A}{\Gamma, A} \mathrm{C} & \frac{\overline{\Gamma^{\rightarrow}, \bar{A}, B}}{\overline{\Gamma^{-}}, A \rightarrow B} \rightarrow^{\mathrm{E}} & \frac{\Gamma}{\Gamma, A} \mathrm{~W} \\
& \frac{\Gamma, A \quad \bar{A}, \Delta}{\Gamma, \Delta} \text { cut } &
\end{array}
$$

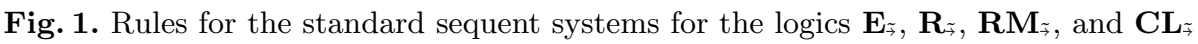

\section{Sequent Calculus, Part II}

In this section we consider formulas in negation normal form (NNF), i.e., the class $\mathcal{L}$ of formulas (also denoted by $A, B, \ldots$ ) generated by the countable set $\mathcal{A}=\{a, b, \ldots\}$ of propositional variables, their duals $\overline{\mathcal{A}}=\{\bar{a}, \bar{b}, \ldots\}$, and the binary connectives $\wedge$ and $\vee$, via the following grammar:

$$
A, B::=a|\bar{a}| A \wedge B \mid A \vee B
$$

An atom is a formula $a$ or $\bar{a}$ with $a \in \mathcal{A}$. As before, a sequent $\Gamma$ is a multiset of formulas separated by comma. We define negation as a function on all formulas in NNF via the De Morgan laws:

$$
\overline{\bar{a}}=a \quad \overline{A \wedge B}=\bar{A} \vee \bar{B} \quad \overline{A \vee B}=\bar{A} \wedge \bar{B}
$$

There is a correspondence between the class $\mathcal{I}$ defined in the previous section and the class $\mathcal{L}$ of formulas in NNF, defined via the two translations $[\cdot]_{\mathcal{L}}: \mathcal{I} \rightarrow \mathcal{L}$ and $[\cdot]_{\mathcal{I}}: \mathcal{L} \rightarrow \mathcal{I}:$

$$
[a]_{\mathcal{L}}=a, \quad[\bar{A}]_{\mathcal{L}}=\overline{[A]_{\mathcal{L}}}, \quad[A \rightarrow B]_{\mathcal{L}}=\overline{[A]_{\mathcal{L}}} \vee[B]_{\mathcal{L}}
$$

and

$$
[a]_{\mathcal{I}}=a,[\bar{a}]_{\mathcal{I}}=\bar{a},[A \vee B]_{\mathcal{I}}=\overline{[A]_{\mathcal{I}}} \rightarrow[B]_{\mathcal{I}},[A \wedge B]_{\mathcal{I}}=\overline{[A]_{\mathcal{I}} \rightarrow \overline{[B]_{\mathcal{I}}}}
$$

Proposition 3.1 If $A$ is a formula in $N N F$, then $\left[[A]_{\mathcal{I}}\right]_{\mathcal{L}}=A$.

The proof is straightforward, but in general we do not have $\left[[B]_{\mathcal{L}}\right]_{\mathcal{I}}=B$ for formulas in $\mathcal{I}$, since we can have arbitrary nestings of negation and $[\bar{B}]_{\mathcal{L}}=[B]_{\mathcal{L}}$. For this reason, we use here the NNF notation, as it is more concise and carries less redundancy.

We can use this correspondence to translate the sequent systems in Figure 1 into the NNF notation. We go one step further and give cut-free systems $\mathrm{LE}^{\prime}$, $\mathrm{LR}^{\prime}, \mathrm{LRM}^{\prime}$, and LK. They are given in Figure 2, where we denote by $\Gamma^{\wedge}$ a sequent of the form $A_{1} \wedge B_{1}, \ldots, A_{n} \wedge B_{n}$ (i.e., all formulas in $\Gamma^{\wedge}$ are conjunctions). 
On Combinatorial Proofs for Logics of Relevance and Entailment

\begin{tabular}{c|c}
\multicolumn{1}{c|}{ MLL } & $a x, \vee, \wedge$ \\
\hline $\mathrm{MLL}_{\text {mix }}$ & ax, $\vee, \wedge$, mix \\
\hline $\mathrm{MLL}_{\mathrm{E}}$ & $\mathrm{ax}, \wedge, \vee \mathrm{E}$ \\
\hline $\mathrm{LE}^{\prime}$ & $\mathrm{ax}, \wedge, \vee \mathrm{E}, \mathrm{C}$ \\
\hline $\mathrm{LR}^{\prime}$ & $\mathrm{ax}, \vee, \wedge, \mathrm{C}$ \\
\hline $\mathrm{LRM}^{\prime}$ & $\mathrm{ax}, \vee, \wedge, \mathrm{C}, \mathrm{mix}$ \\
\hline $\mathrm{LK}$ & $\mathrm{ax}, \vee, \wedge, \mathrm{C}, \mathrm{W}$
\end{tabular}

$$
\begin{array}{cll}
\frac{}{a, \bar{a}} \text { ax } & \frac{\Gamma^{\wedge}, A, B}{\Gamma^{\wedge}, A \vee B} \vee \mathrm{E} & \frac{\Gamma, A, B}{\Gamma, A \vee B} \vee \\
\frac{\Gamma, A, A}{\Gamma, A} \mathrm{C} & \frac{\Gamma, A \Delta, B}{\Gamma, \Delta, A \wedge B} \wedge \\
\frac{\Gamma}{\Gamma, A} \mathrm{~W} & \frac{\Gamma \Delta}{\Gamma, \Delta} \text { mix }
\end{array}
$$

Fig. 2. The cut-free sequent systems for formulas in NNF

$$
\frac{\Gamma\{(A \vee B) \wedge(C \vee D)\}}{\Gamma\{(A \vee C) \vee(B \vee D)\}} \mathrm{m}^{\downarrow} \quad \frac{\Gamma\{A \vee A\}}{\Gamma\{A\}} \mathrm{C}^{\downarrow} \quad \frac{\Gamma\{a \vee a\}}{\Gamma\{a\}} \mathrm{ac}^{\downarrow} \quad \frac{\Gamma\{B\}}{\Gamma\{B \vee A\}} \mathrm{W}^{\downarrow}
$$

Fig. 3. The deep rules for medial, contraction, atomic contraction and weakening.

That figure also defines the linear logic systems $M L L_{E}$ and $M L L$ that we will need in the course of this paper.

We make also use of the deep inference rules in Figure 3 (see also [106]), where a context $\Gamma\{\}$ is a sequent or a formula, where a hole \{\} takes the place of an atom. We write $\Gamma\{A\}$ when we replace the hole with a formula $A$.

If $\mathrm{S}$ is a sequent system and $\Gamma$ a sequent, we write $\stackrel{\mathrm{S}}{\longleftarrow} \Gamma$ if $\Gamma$ is derivable in $\mathrm{S}$. Moreover, if $\mathrm{S}$ is a set of inference rules with exactly one premise, we write $\Gamma^{\prime} \stackrel{\mathrm{S}}{\longmapsto} \Gamma$ whenever there is a derivation from $\Gamma^{\prime}$ to $\Gamma$ using only rules in S.

Theorem 3.2 If $\Gamma$ is a sequent in $\mathcal{L}$, then

$$
\stackrel{\mathrm{LE}^{\prime} \cup\{\text { cut }\}}{\longmapsto} \Gamma \quad \Longleftrightarrow \quad \stackrel{\mathrm{LE}_{\Im}}{\longmapsto}[\Gamma]_{\mathcal{I}}
$$

Proof The proof follows the definitions of the two translations $[\cdot]_{\mathcal{I}}$ and $[\cdot]_{\mathcal{L}}$. In fact, C- and cut-rules are the same in the two systems and and $\rightarrow$-rule is equivalent to $\vee_{\mathrm{E}}$-rule. Moreover, it $\mathrm{s}$ trivial to prove by induction that $\stackrel{\mathrm{LE}^{\prime}}{\longmapsto} A, \bar{A}$. Finally:

$$
\frac{\mathrm{LE}^{\prime} \|}{\bar{A}, \overline{A \rightarrow B}, B} \mathrm{EAX} \leadsto \frac{[\bar{A}]_{\mathcal{L}},[A]_{\mathcal{L}}[\bar{B}]_{\mathcal{L}},[B]_{\mathcal{L}}}{[\bar{A}]_{\mathcal{L}},\left([A]_{\mathcal{L}} \wedge[\bar{B}]_{\mathcal{L}}\right),[B]_{\mathcal{L}}} \wedge \mathrm{E}
$$

and

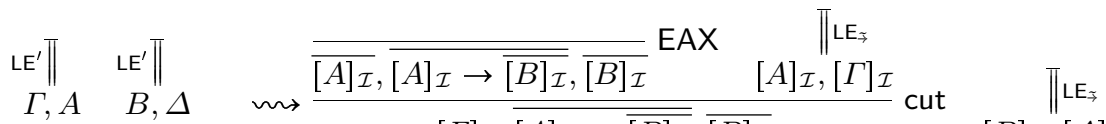

$$
\begin{aligned}
& \frac{\Gamma, A \quad B, \Delta}{\Gamma, A \wedge B, \Delta} \wedge \mathrm{E} \leadsto \frac{[\Gamma]_{\mathcal{I}}, \overline{[A]_{\mathcal{I}} \rightarrow \overline{[B]_{\mathcal{I}}}, \overline{[B]_{\mathcal{I}}}} \text { cut } \quad{ }^{\left[\mathrm{LE}_{\mathcal{I}}\right.}}{[B]_{\mathcal{I}},[\Delta]_{\mathcal{I}}} \text { cut } \\
& {[\Gamma]_{\mathcal{I}}, \overline{[A]_{\mathcal{I}} \rightarrow \overline{[B]_{\mathcal{I}}}},[\Delta]_{\mathcal{I}}}
\end{aligned}
$$


One important property of the systems in Figure 2 is cut admissibility.

Theorem 3.3 (Cut admissibility) Let $\Gamma$ be a sequent in $\mathcal{L}$, and let $\mathrm{S}$ be one of the systems $\mathrm{MLL}, \mathrm{MLL}_{\text {mix }}, \mathrm{MLL}_{\mathrm{E}}, \mathrm{LE}^{\prime}, \mathrm{LR}^{\prime}, \mathrm{LRM}^{\prime}, \mathrm{LK}$. Then

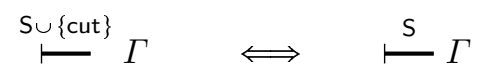

Proof The proof is a standard cut permutation argument. For LK it can already be found in [9] and for all other systems it is the same proof, observing that no reduction step introduces a rule that is not present in the system.

The following lemma relates the mix-rule from linear logic to the mingle axiom rule $\mathrm{mAX}$ :

Lemma 3.4 Let $\mathrm{S}$ be a sequent system, if $\Gamma$ is a sequent in $\mathcal{L}$ then

$$
\stackrel{\mathrm{S} \cup\{\operatorname{mAX}\}}{ } F \Longleftrightarrow \stackrel{\mathrm{S} \cup\{\operatorname{mix}\}}{ } F
$$

Proof First, mAX can be derived using mix:

$$
\overline{A_{1}, \ldots, A_{n}, \bar{A}_{1}, \ldots, \bar{A}_{n}} \operatorname{mAX} \leadsto \begin{gathered}
\mathrm{s}\|\mathrm{s}\| \\
\frac{A_{1}, \bar{A}_{1} \quad A_{2}, \bar{A}_{2}}{A_{1}, \bar{A}_{1}, A_{2}, \bar{A}_{2}} \text { mix } \\
\frac{A_{1}, \bar{A}_{1}, \ldots, A_{n-1}, \bar{A}_{n-1} \quad A_{n}, \bar{A}_{n}}{A_{1}, \bar{A}_{1}, \ldots, A_{n}, \bar{A}_{n}} \operatorname{mix}
\end{gathered}
$$

Conversely, if $\Gamma, \Delta$ is the conclusion of a mix inference,

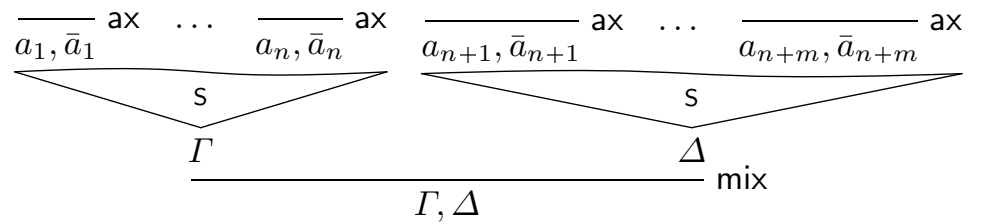

it suffices to replace one axiom of the derivation of $\Gamma$ and one axiom of the derivation of $\Delta$ by a single $\mathrm{mAX}$, that is

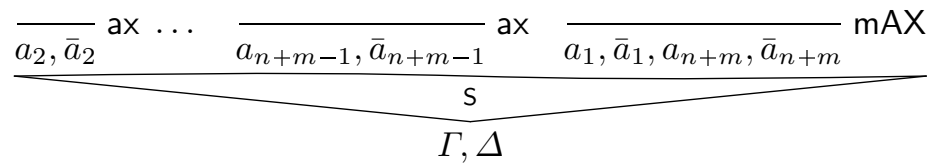

This is enough to show the equivalence between the systems in Figures 1 and 2 
Theorem 3.5 If $\Gamma$ is a sequent in $\mathcal{L}$, then

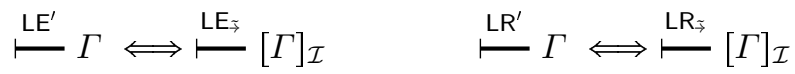

$$
\begin{aligned}
& \stackrel{\mathrm{LRM}^{\prime}}{\longmapsto} \Gamma \Longleftrightarrow \stackrel{\mathrm{LRM}_{\mp}}{\longmapsto}[\Gamma]_{\mathcal{I}} \quad \stackrel{\mathrm{LK}}{=} \Gamma \Longleftrightarrow \stackrel{\mathrm{LK}_{\mp}}{\longleftarrow}[\Gamma]_{\mathcal{I}}
\end{aligned}
$$

Proof This follows from Theorems 3.2 and 3.3 and Lemma 3.4 , using the definitions of $[\cdot]_{\mathcal{I}}$ and $[\cdot]_{\mathcal{L}}$.

Finally, the most important reason to use the systems in Figure 2 instead of the ones in Figure 1 is the following decomposition theorem:

Theorem 3.6 If $\Gamma$ is a sequent in $\mathcal{L}$, then

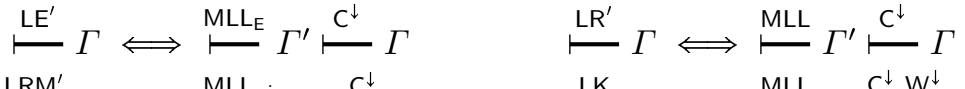

$$
\begin{aligned}
& \stackrel{\mathrm{LRM}^{\prime}}{\longmapsto} \Gamma \Longleftrightarrow \stackrel{\mathrm{MLL}_{\text {mix }}}{\longmapsto} \Gamma^{\prime} \stackrel{\mathrm{C}^{\downarrow}}{\longmapsto} \Gamma \quad \stackrel{\mathrm{LK}}{\longleftarrow} \Gamma \Longleftrightarrow \stackrel{\mathrm{MLL}}{\longmapsto} \Gamma^{\prime} \stackrel{\mathrm{C}^{\downarrow}, \mathrm{W}^{\downarrow}}{\longmapsto} \Gamma
\end{aligned}
$$

Proof The proof is given by rules permutation. It suffices to consider all W- and C-rules as their deep counterpart and move their instance as down as possible in the derivation. Conversely, it suffices to move up all occurrences of $C^{\downarrow}$ and $W \downarrow$ until the context is shallow and then replace them by $\mathrm{C}$ and $\mathrm{W}$ instances.

\section{Cographs}

A graph $\mathcal{G}=\left\langle V_{\mathcal{G}}, \stackrel{\mathcal{G}}{=}\right\rangle$ is a set $V_{\mathcal{G}}$ vertices and a set $\mathscr{\mathcal { G }}$ of edges, which are two-

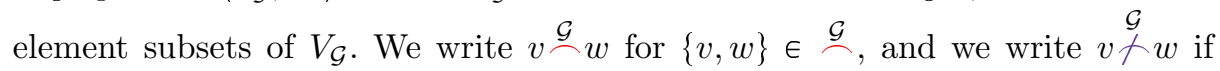
$\{v, w\} \notin \mathcal{G}$. We omit the index/superscript $\mathcal{G}$ when it is clear from the context. When drawing a graph we use $v-w$ for $v \frown w$. If $v \nmid w$ and $v \neq w$ we either draw no edge or use $v-w$.

A cograph $\mathcal{G}$ is a $\mathrm{P} 4$-free graph, i.e. a graph $\mathcal{G}$ with no $u, v, y, z \in V$ such that their induced subgraph has the following shape 4

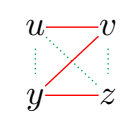

For two disjoint graphs $\mathcal{G}$ and $\mathcal{H}$, we define their (disjoint) union $\mathcal{G} \vee \mathcal{H}$ and their join $\mathcal{G} \wedge \mathcal{H}$ as follows:

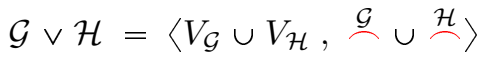

$$
\begin{aligned}
& \mathcal{G} \wedge \mathcal{H}=\left\langle V_{\mathcal{G}} \cup V_{\mathcal{H}}, \stackrel{\mathcal{G}}{\sim} \stackrel{\mathcal{H}}{\sim} \cup\left\{\{u, v\} \mid u \in V_{\mathcal{G}}, v \in V_{\mathcal{H}}\right\}\right\rangle
\end{aligned}
$$

\footnotetext{
${ }^{4}$ In the literature, this condition is also called Z-free or N-free.
} 
which can be visualized as follows:
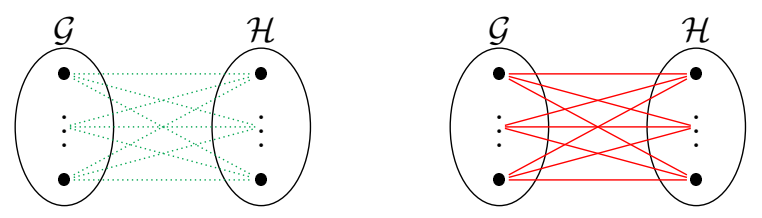

We say that a graph is $\mathcal{A}$-labeled if each vertex is marked with an atom in $\mathcal{A} \cup \overline{\mathcal{A}}$. We can associate to each formula $F$ in $\mathcal{L}$ an $\mathcal{A}$-labeled cograph $\llbracket F \rrbracket$ inductively:

$$
\llbracket a \rrbracket=\bullet_{a}, \quad \llbracket \bar{a} \rrbracket=\bullet_{\bar{a}}, \quad \llbracket A \vee B \rrbracket=\llbracket A \rrbracket \vee \llbracket B \rrbracket, \quad \llbracket A \wedge B \rrbracket=\llbracket A \rrbracket \wedge \llbracket B \rrbracket
$$

If $\Gamma=A_{1}, \ldots, A_{n}$ is a sequent of formulas in $\mathcal{L}$, we define $\llbracket \Gamma \rrbracket=\llbracket A_{1} \rrbracket \vee \cdots \vee \llbracket A_{n} \rrbracket$.

The interest in cographs comes from the following two well-known theorems (see, e.g., [814]).

Theorem 4.1 $A \mathcal{A}$-labeled graphs $\mathcal{G}$ is a cograph iff there is a formula $F \in \mathcal{L}$ such that $\mathcal{G}=\llbracket F \rrbracket$.

Theorem $4.2 \llbracket F \rrbracket=\llbracket F^{\prime} \rrbracket$ iff $F$ and $F^{\prime}$ are equivalent modulo associativity and commutativity of $\wedge$ and $\vee$.

\section{Skew fibrations}

Definition 5.1 Let $\mathcal{G}$ and $\mathcal{H}$ be graphs. A skew fibration $f: \mathcal{G} \rightarrow \mathcal{H}$ is a mapping from $V_{\mathcal{G}}$ to $V_{\mathcal{H}}$ that preserves $\frown$ :

- if $u \stackrel{\mathcal{G}}{\stackrel{5}{v}}$ then $f(u) \stackrel{\mathcal{H}}{-} f(v)$,

and that has the skew lifting property:

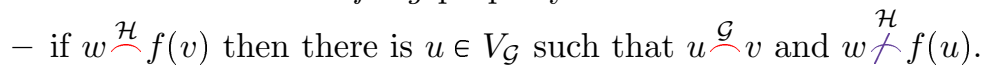

A skew fibration $f: \mathcal{G} \rightarrow \mathcal{H}$ is relevant if it is surjective on vertices and on $\frown$ :

- for every $w \in V_{\mathcal{H}}$ there is a $u \in V_{\mathcal{G}}$ such that $f(u)=w$, and

- if $w \stackrel{\mathcal{H}}{\stackrel{4}{t}}$ then there are $u, v \in V_{\mathcal{G}}$ such that $f(u)=w$ and $f(v)=t$ and $u \stackrel{\mathcal{G}}{ } v$.

The purpose of skew fibrations in this setting is to give a combinatorial characterization of derivations containing only contractions and weakenings.

Theorem 5.2 If $\Gamma, \Gamma^{\prime}$ are sequents in $\mathcal{L}$ then

1. $\Gamma^{\prime} \stackrel{C^{\downarrow}, \mathrm{W}^{\downarrow}}{\mathrm{C}^{\downarrow}} \Gamma$ iff there is a skew fibration $f: \llbracket \Gamma^{\prime} \rrbracket \rightarrow \llbracket \Gamma \rrbracket$.

2. $\Gamma^{\prime} \stackrel{\mathrm{C}^{\downarrow}}{\longmapsto} \Gamma$ iff there is a relevant skew fibration $f: \llbracket \Gamma^{\prime} \rrbracket \rightarrow \llbracket \Gamma \rrbracket$. 
Proof The first statement has been proved independently in [12] and in [16].

The proof of the second statement is similar, but relevant condition rules out weakening. Let $\Gamma^{\prime}=\Gamma_{0}, \Gamma_{1}, \ldots, \Gamma_{n}=\Gamma$ such that $\frac{\Gamma_{i}\left\{A_{i} \vee A_{i}\right\}}{\Gamma_{i+1}=\Gamma_{i}\left\{A_{i}\right\}} \mathrm{C}^{\downarrow}$. By induction over the size of $A_{i}$, there is a relevant skew fibration $f_{i}: \Gamma_{i} \rightarrow \Gamma_{i+1}$ for each $i \in\{0, \ldots n-1\}$ and the composition of such $f_{i}$ is still a relevant skew fibration. Conversely, in case of $f$ a relevant skew fibration, the lifting property becomes the following:

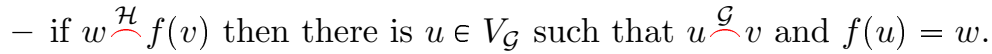

which, by induction over $\Gamma$, allows to prove that:

- if $\llbracket \Gamma \rrbracket=\bullet_{a}$ then $\llbracket \Gamma^{\prime} \rrbracket=\bullet_{a} \vee \cdots \vee \bullet_{a}$;

- if $\llbracket \Gamma \rrbracket=\mathcal{G} \vee \mathcal{H}$ then $\llbracket \Gamma^{\prime} \rrbracket=\mathcal{G}^{\prime} \vee \mathcal{H}^{\prime}$ with $f\left(\mathcal{G}^{\prime}\right)=\mathcal{G}$ and $f\left(\mathcal{H}^{\prime}\right)=\mathcal{H}$;

- if $\llbracket \Gamma \rrbracket=\mathcal{G} \wedge \mathcal{H}$ then either $\llbracket \Gamma^{\prime} \rrbracket=\mathcal{G}^{\prime} \wedge \mathcal{H}^{\prime}$ with $f\left(\mathcal{G}^{\prime}\right)=\mathcal{G}$ and $f\left(\mathcal{H}^{\prime}\right)=\mathcal{H}$, or $\llbracket \Gamma^{\prime} \rrbracket=\left(\mathcal{G}_{1}^{\prime} \wedge \mathcal{H}_{1}^{\prime}\right) \vee \cdots \vee\left(\mathcal{G}_{n}^{\prime} \wedge \mathcal{H}_{n}^{\prime}\right)$ with $f\left(\mathcal{G}_{i}^{\prime}\right)=\mathcal{G}$ and $f\left(\mathcal{H}_{i}^{\prime}\right)=\mathcal{H}$ for each $i \in\{1, \ldots, n\}$.

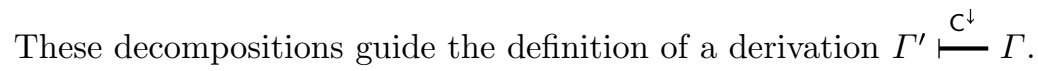

\section{RB-cographs and combinatorial proofs}

In this section we finally define combinatorial proofs. For this we use Retoré's RB-cographs [15]:

Definition 6.1 ([15]) An $R B$-cograph is a tuple $\mathcal{G}=\left\langle V_{\mathcal{G}}, \stackrel{\mathcal{G}}{\stackrel{\mathcal{G}}{\vee}\rangle}\right.$ where $\mathcal{G}^{R}=$ $\left\langle V_{\mathcal{G}}, \stackrel{\mathcal{G}}{\neg}\right\rangle$ is a cograph and $\mathcal{G}$ a irreflexive, symmetric binary relation such that for every $v \in V_{\mathcal{G}}$ there is a unique $w \in V_{\mathcal{G}}$ with $v \stackrel{\mathcal{G}}{\vee} w$.

As done in (1) in the introduction, we use $v-w$ for $v \frown w$, and $v-w$ for $v \vee w$ when drawing an RB-cograph.

Definition 6.2 ([15]) If $u$ and $v$ are two vertices of a RB-cograph, an alternating elementary path (a-path) from $x_{0}$ to $x_{n}$ is a sequence of pairwise disjoint vertices $x_{0}, \ldots, x_{n} \in V$ such that either $x_{0} \frown x_{1} \vee x_{2} \frown x_{3} \vee x_{4} \cdots x_{n}$ or $x_{0} \vee x_{1} \frown x_{2} \vee x_{3} \frown x_{4} \cdots x_{n}$. An $a$-cycle is an $æ$-path of even length with $x_{0}=$ $x_{n}$. A chord of æ-path $x_{0}, \ldots, x_{n}$ is an edge $x_{i} \frown x_{j}$ with $i+1<j$. The æ-path is chordless if it has no chord. A RB-cograph is a-connected if there is a chordless æ-path between each pair of vertices $\mathcal{G}$ and it is $\mathfrak{x}$-acyclic if there are no chordless æ-cycle.

Theorem 6.3 ([15]) If $\Gamma$ is a sequent over $\mathcal{L}$ then
1. $\stackrel{\mathrm{MLL}}{\longmapsto} \Gamma \Longleftrightarrow$ there is an $\mathfrak{}$-connected, a-acyclic $R B$-cograph $\mathcal{G}$ with $\mathcal{G}^{R}=\llbracket \Gamma \rrbracket$
2. $\stackrel{\mathrm{MLL}_{\text {mix }}}{\longmapsto} \Gamma \Longleftrightarrow$ there is an a-acyclic RB-cograph $\mathcal{G}$ with $\mathcal{G}^{R}=\llbracket \Gamma \rrbracket$ 
We say that a map $f$ from an RB-cograph $\mathcal{C}$ to a $\mathcal{A}$-labeled cograph is axiom preserving if for all $u, v$ with $u \stackrel{\mathcal{C}}{v} v$ we have that $f(u)$ and $f(v)$ are labeled by two dual atoms.

Definition 6.4 Let $\Gamma$ be a sequent over $\mathcal{L}$.

1. A combinatorial LK-proof of $\Gamma$ is an axiom-preserving skew fibration $f: \mathcal{C} \rightarrow$ $\llbracket \Gamma \rrbracket$ where $\mathcal{C}$ is an $æ$-connected, $æ$-acyclic RB-cograph.

2. A combinatorial $\mathrm{LR}^{\prime}$-proof of $\Gamma$ is an is an axiom-preserving relevant skew fibration $f: \mathcal{C} \rightarrow \llbracket \Gamma \rrbracket$ where $\mathcal{C}$ is an æ-connected, æ-acyclic RB-cograph.

3. Finally, a combinatorial $\mathrm{LRM}^{\prime}$-proof of $\Gamma$ is an is an axiom-preserving relevant skew fibration $f: \mathcal{C} \rightarrow \llbracket I \rrbracket$ where $\mathcal{C}$ is an $æ$-acyclic RB-cograph.

Theorem 6.5 Let $\Gamma$ be a sequent over $\mathcal{L}$, and let $\mathrm{S} \in\left\{\mathrm{LR}^{\prime}, \mathrm{LRM}^{\prime}, \mathrm{LK}\right\}$. Then

$$
\stackrel{\mathrm{S}}{\mathrm{S}} \Longleftrightarrow \text { there is a combinatorial S-proof of } \Gamma \text {. }
$$

Proof This follows from Theorems 3.6, 5.2 and 6.3. For LK this has already been shown in [1112 16 17].

Below are a combinatorial LR'-proof (on the left) and a combinatorial LRM'proof (on the right):
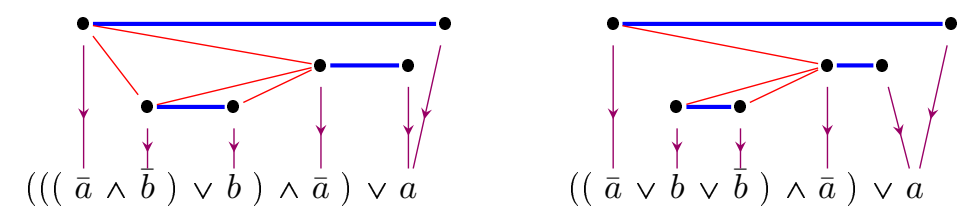

Theorem 6.6 Let $\Gamma$ be a sequent and $\mathcal{G}$ a graph together with a perfect matching, and let $f$ be a map from $\mathcal{G}$ to $\llbracket \Gamma \rrbracket$. It can be decided in polynomial time in $\left|V_{\mathcal{G}}\right|+|\Gamma|$ whether $f: \mathcal{G} \rightarrow \llbracket \Gamma \rrbracket$ is a combinatorial $\mathrm{LR}^{\prime}$-proof (resp. a combinatorial $\mathrm{LRM}^{\prime}$-proof).

Proof All necessary properties can be checked in polynomial time.

\section{Sequent Calculus, Part III}

In the remainder of the paper, we extend our results to the entailment logic $\mathbf{E}_{\mathfrak{7}}$. The reason why we need a separate treatment is due to some intrinsic technical drawbacks occurring in the $L^{\prime}$ sequent calculus. The first is that commas used to separate formulas in a sequent can not be interpreted as disjunction, as we usually do in classical logic. Using the display calculi 4 terminology, in $\mathrm{LE}^{\prime}$ the comma is extensional while $\vee$ and $\wedge$ are intensional. Moreover, $\wedge$ and $\vee$ are not associative and this give birth to unusual behaviors. For example $(A \vee A) \vee(\bar{A} \wedge \bar{A})$ is provable in $\mathrm{LE}^{\prime}$ while $A \vee(A \vee(\bar{A} \wedge \bar{A}))$ is not. 


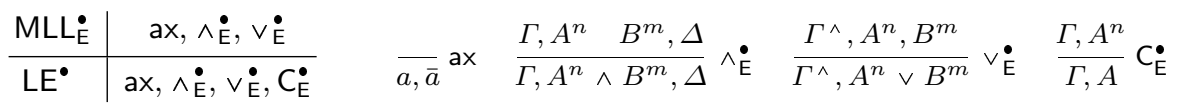

Fig. 4. The cut-free systems $\mathrm{MLL}_{\mathrm{E}}^{\bullet}$ and $\mathrm{LE}^{\bullet}$

We first introduce the class of entailed formulas $\mathcal{E}$ which are generated by a countable set $\mathcal{A}=\{a, b, \ldots\}$ of propositional variables and the following grammar:

$$
A, B::=a|\bar{a}| A \wedge B|A \vee B| A^{n}
$$

where $n>0$. Moreover, we consider the sequents $\Gamma\left\{A^{n+1}\right\}$ and $\Gamma\left\{A, A^{n}\right\}$ to be equal. In other words, $A^{n}$ has to be thought of as an abbreviation for the sequent $A, \ldots, A$ ( $n$ copies of $A$ ) that is allowed to occur as a subformula in a formula. We define the sequent systems $\mathrm{MLL}_{\mathrm{E}}$ and $\mathrm{LE}^{\bullet}$ on entailed formulas given by the rules in Figure 4 .

Theorem 7.1 If $\Gamma$ is a sequent over $\mathcal{L}$ then

$$
\stackrel{\mathrm{LE}^{\prime}}{\longmapsto} \Gamma \stackrel{\mathrm{LE}^{\bullet}}{\longmapsto} \Gamma
$$

Proof It suffices to remark that $\mathrm{LE}^{\bullet}$ rules behave as $\mathrm{LE}^{\prime}$ rules on standard NNFformulas. Let $\mathrm{C}_{\mathrm{E}}^{\downarrow}$ be the deep inference rule $\frac{F\left\{A^{n}\right\}}{F\{A\}} \mathrm{C}_{\mathrm{E}}^{\downarrow}$. Then we have a result similar
to Theorem 3.6.

Theorem 7.2 If $F$ is a formula in $\mathcal{E}$ then

$$
\stackrel{\mathrm{LE}^{\bullet}}{\longmapsto} F \quad \Longleftrightarrow \quad \stackrel{\mathrm{MLL}_{\mathrm{E}}^{\bullet}}{\longmapsto} F^{\prime} \stackrel{\mathrm{C}_{\mathrm{E}}^{\downarrow}}{\longmapsto} \Gamma
$$

Proof By rule permutations, similarly to the proof of Theorem 3.6 .

\section{Weighted cographs and fibrations}

Definition 8.1 A weighted graph $\mathcal{G}=\left\langle V_{\mathcal{G}}, \stackrel{\mathcal{G}}{\underline{1}}, \delta\right\rangle$ is a given by graph $\left\langle V_{\mathcal{G}}, \stackrel{\mathcal{G}}{ }\right\rangle$ together with a weight function $\delta: V_{\mathcal{G}} \times V_{\mathcal{G}} \rightarrow \mathbb{N}$ such that if $u \stackrel{\mathcal{G}}{v}$ then $\delta(u, v)>0$ and $\delta(u, u)=0$.

We use the following notations: we write $u \stackrel{\mathcal{G}}{~}_{k} v$ iff $u \stackrel{\mathcal{G}}{v}$ and $\delta(u, v)=k$, and we write $u \mathcal{G}_{k} v$ iff $u \stackrel{\mathcal{G}}{\dagger} v$ and $\delta(u, v)=k$. When drawing a graph we use $v-k-w$ for $v{ }_{k} w$ and we use $v \cdots \cdots \cdot k \cdots w$ for $v \smile_{k} w$. If $v \smile_{0} w$ we often draw no edges.

Definition 8.2 A weighted cograph is a weighted graph such that:

1. the graphs $\left\langle V_{\mathcal{G}}, \stackrel{\mathcal{G}}{i}_{i}\right\rangle$ and $\langle V_{\mathcal{G}}, \underbrace{}_{i}\rangle$ are Z-free for all $i \neq 0$; 
2. for all $u, v, w \in V_{\mathcal{G}}$, and any $n, m, k, l, h \in \mathbb{N}$, with $n, m, k$ being pairwise distinct and $h>0$, the following configurations are forbidden:

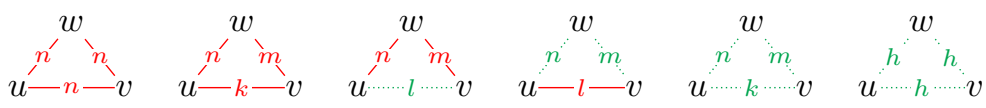

3. for all $u, v, w \in V_{\mathcal{G}}$ with

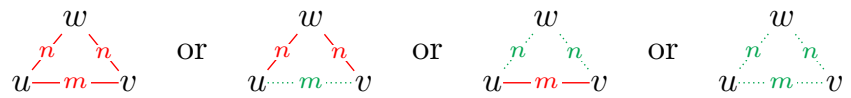

either $n=0$ or $m=0$ or $n>m$.

Remark 8.3 A cograph is a weighted cograph $\mathcal{G}$ with $\delta: V_{\mathcal{G}} \times V_{\mathcal{G}} \rightarrow\{0,1\}$.

We define the juxtaposition, graded union and graded join operations:

$$
\begin{aligned}
& \mathcal{G} \star \mathcal{H}=\left\langle V_{\mathcal{G}} \cup V_{\mathcal{H}}, \stackrel{\mathcal{G}}{\mathcal{G}} \cup \frac{\mathcal{H}}{\mathcal{H}}, \delta_{\mathcal{G}} \cup \delta_{\mathcal{H}} \cup \delta^{0}\right\rangle \\
& \mathcal{G} \vee \mathcal{H}=\left\langle V_{\mathcal{G}} \cup V_{\mathcal{H}}, \stackrel{\mathcal{G}}{\mathcal{H}}, \delta_{\mathcal{G}} \cup \delta_{\mathcal{H}} \cup \delta^{\smile}\right\rangle \\
& \mathcal{G} \wedge \mathcal{H}=\left\langle V_{\mathcal{G}} \cup V_{\mathcal{H}}, \stackrel{\mathcal{G}}{\mathcal{H}} \cup\{\{u, v\} \mid u \in \mathcal{G}, v \in \mathcal{H}\}, \delta_{\mathcal{G}} \cup \delta_{\mathcal{H}} \cup \delta^{\frown}\right\rangle
\end{aligned}
$$

where $\delta^{0}$ is the weight function which assigns to each $(u, v) \in V_{\mathcal{G}} \times V_{\mathcal{H}} \cup V_{\mathcal{H}} \times V_{\mathcal{G}}$ the weight 0 , while $\delta^{\frown}$ (resp. $\delta^{-}$) is the weight function which assigns to each $(u, v) \in V_{\mathcal{G}} \times V_{\mathcal{H}} \cup V_{\mathcal{H}} \times V_{\mathcal{G}}$ the weight $k=1+\max \{\delta(w, z) \mid w \stackrel{\mathcal{G}}{z} z$ or $w \stackrel{\mathcal{H}}{=} z\}$ (respectively $k=1+\max \{\delta(w, z) \mid w \nmid \mathcal{\mathcal { G }} z$ or $w \nmid z\})$. We represent these operations as follows:
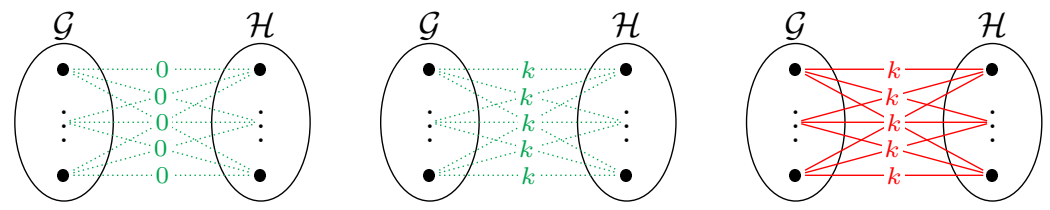

We associate to each entailed formula $F$ (sequent $\Gamma$ ) a graded relation web:

$$
\begin{gathered}
\llbracket a \rrbracket=\bullet_{a}, \quad \llbracket \bar{a} \rrbracket=\bullet_{\bar{a}}, \quad \llbracket A \vee B \rrbracket=\llbracket A \rrbracket \vee \llbracket B \rrbracket, \\
\llbracket A \wedge B \rrbracket=\llbracket A \rrbracket \wedge \llbracket B \rrbracket, \quad \llbracket A, B \rrbracket=\llbracket A \rrbracket \star \llbracket B \rrbracket
\end{gathered}
$$

Two weighted graphs $\mathcal{G}$ and $\mathcal{H}$ are isomorphic (denoted $\mathcal{G} \simeq \mathcal{H}$ ) if there is a bijection $\phi$ between $V_{\mathcal{G}}$ and $V_{\mathcal{H}}$ which preserves edges and weights order, that

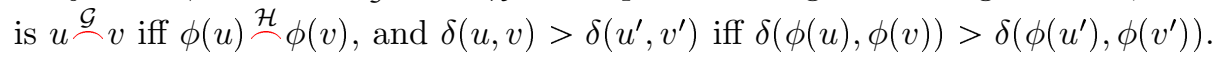
Then Theorem 4.1 can be extended to the following:

Theorem 8.4 A $\mathcal{A}$-labeled weighted graph $\mathcal{G}$ is a weighted cograph iff there is a sequent $\Gamma$ of entailed formulas such that $\mathcal{G} \simeq \llbracket \Gamma \rrbracket$. 
Proof The proof is similar to the one of Theorem 4.1. However, the condition $\mathcal{G} \simeq \llbracket \Gamma \rrbracket$ (instead of $\mathcal{G}=\llbracket \Gamma \rrbracket)$ is due to the existence of weighted cographs not of the form $\llbracket \Gamma \rrbracket$. By means of example take $a-2-b \simeq \llbracket a \wedge b \rrbracket=a-1-b$.

Definition 8.5 A weighted skew fibration $f: \mathcal{G} \rightarrow \mathcal{H}$ is a skew fibration between weighted graphs that preserves the weights.

Note that this means in particular that $f(u)=f(v)$ implies that $\delta(u, v)=0$. Theorem 8.6 Let $\Gamma$ and $\Gamma^{\prime}$ be sequents over $\mathcal{E}$. Then $\Gamma^{\prime} \stackrel{\mathrm{C}_{\mathrm{E}}^{\downarrow}}{\longrightarrow} \Gamma$ iff there is a weighted relevant skew fibration $f: \llbracket \Gamma^{\prime} \rrbracket \rightarrow \llbracket \Gamma \rrbracket$.

Proof The proof is similar to the one for (non-weighted) relevant skew fibrations. First, let $\Gamma^{\prime}=\Gamma_{0}, \Gamma_{1}, \ldots, \Gamma_{n}=\Gamma$ be a sequence of sequents such that

$$
\frac{\Gamma_{i}\left\{A_{i}, A_{i}\right\}}{\Gamma_{i+1}=\Gamma_{i}\left\{A_{i}\right\}} \mathrm{C}_{\mathrm{E}}^{\downarrow}
$$

By definition of juxtaposition, join and union cograph operations we have that $f_{i}: \llbracket \Gamma_{i} \rrbracket \rightarrow \llbracket \Gamma_{i+1} \rrbracket$ is a relevant skew fibration and preserves $\frown$ and weights. Then also $f=f_{n-1} \circ \cdots \circ f_{0}$ is a weighted relevant skew fibration.

The converse follows by remarking that $f(u)=f(v)$ iff $u \smile_{0} v$.

\section{$9 \quad$ Weighted RB-cographs}

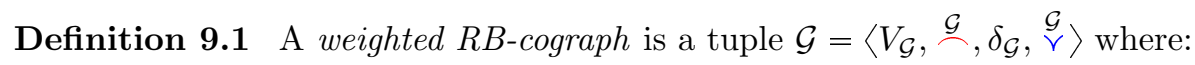

$-\mathcal{G}^{R \delta}=\left\langle V_{\mathcal{G}}, \stackrel{\mathcal{G}}{\lessgtr}, \delta_{\mathcal{G}}\right\rangle$ is a weighted cograph;

$-\stackrel{\mathcal{G}}{\checkmark}$ is a perfect matching on $V_{\mathcal{G}}$;

A weighted RB-cograph $\mathcal{G}=\left\langle V_{\mathcal{G}}, \stackrel{\mathcal{G}}{\underline{1}}, \delta_{\mathcal{G}}, \stackrel{\mathcal{G}}{\vee}\right\rangle$ is e-connected (á-acyclic) if the RB-cograph $\mathcal{G}^{R B}=\left\langle V_{\mathcal{G}}, \stackrel{\mathcal{G}}{\stackrel{\mathcal{G}}{\vee}\rangle}\right.$ is an æ-connected (æ-acyclic) RB-cograph. A weighted RB-cograph is entailed if it is æ-connected, æ-acyclic, and satisfies the following condition:

- if $a, b, c \in V$ such that $a \smile_{m} b$ for $m>0$, and $c \smile_{n} a$ and $c \smile_{n} b$, with $n>m$ or $n=0$, then there is $d \in V$ such that

$$
\begin{aligned}
& a \ldots m \ldots b \\
& \dot{n} n \\
& c-k-d
\end{aligned}
$$

Theorem 9.2 If $\Gamma$ is a sequent of entailed formulas then

$$
\stackrel{\mathrm{MLL}_{\mathrm{E}}}{\longmapsto} \Gamma \Longleftrightarrow \text { there is an entailed weighted } R B \text {-cograph } \mathcal{G} \text { with } \mathcal{G}^{R \delta}=\llbracket \Gamma \rrbracket
$$

Proof The proof piggybacks on Retoré's sequentialization proof [15]. Each proof in $\mathrm{MLL}_{E}$ induces the construction of an entailed weighted cograph $\mathcal{G}$ by the 


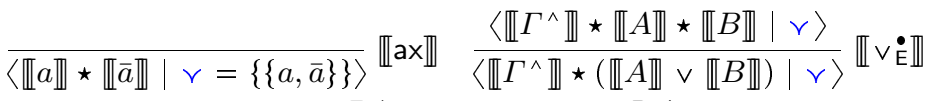

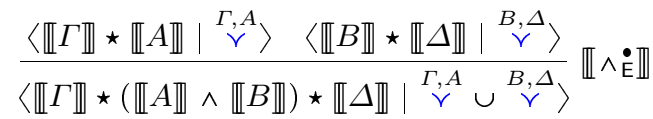

Fig. 5. Construction rules for entailed weighted RB-cographs.

operations shown in Figure 5. In fact, each of these operations preserves æconnectedness, æ-acyclicity and entailment conditions.

Conversely, let $\Gamma$ be the sequent such that $\llbracket \Gamma \rrbracket=\mathcal{G}^{R \delta}$ and let $F_{\Gamma}$ be the formula in $\mathcal{L}$ obtained by substituting each comma occurring in $\Gamma$ by a $\vee$. By Theorem 6.3 we have derivation $\pi_{\mathrm{MLL}}$ of $F_{\Gamma}$ in MLL. We construct a derivation $\pi_{\mathrm{LE}}$ of $\Gamma$ in $\mathrm{MLL}_{\mathrm{E}}$ by induction over the rules in $\pi_{\mathrm{MLL}}$ :

- If the last rule in $\pi_{\mathcal{L}}$ is an ax-rule, then the last rule in $\pi_{\mathrm{LE}}^{\cdot}$ is a ax-rule;

- If the last rule in $\pi_{\mathcal{L}}$ is an $\vee$-rule of the form $\frac{\Gamma, A, B}{\Gamma, A \vee B} \vee$, then $\delta(a, b)=$ $\delta\left(a^{\prime}, b^{\prime}\right)$ for all $a, a^{\prime} \in V_{\mathbb{U}} A \rrbracket$ and $b, b^{\prime} \in V_{\mathbb{U} B \rrbracket}$. If $\delta(u, v)=0$ we skip this rule inference in the construction of $\pi_{\mathrm{LE}}$ (the $\vee$ introduced by this rule in $F_{\Gamma}$ is a comma in $\Gamma$ ). Otherwise, the last rule in $\pi_{\mathrm{LE}}^{\bullet}$ is a $\vee_{\mathrm{E}}^{-}$-rule. In fact, for each $c \in V_{\mathbb{I}} \Gamma \mathbb{}$ by entailment condition there are $d \in V_{\mathbb{I} \Gamma \mathbb{}}$ such that $c \frown d$; that is $\Gamma=\Gamma^{\wedge}$

- If the last rule in $\pi_{\mathcal{L}}$ is an $\wedge$-rule, then the last rule in $\pi_{\mathrm{LE}}^{\bullet}$ is a $\wedge_{\mathrm{E}^{-}}$-rule.

Definition 9.3 A combinatorial LE'-proof of a sequent $\Gamma$ in $\mathcal{L}$ is given by an axiom-preserving weighted relevant skew fibration $f: \mathcal{C}^{R \delta} \rightarrow \llbracket \Gamma \rrbracket$ where $\mathcal{C}$ is an entailed weighted RB-cograph.

Theorem 9.4 Let $\Gamma$ be a sequent in $\mathcal{L}$ then

$$
\stackrel{\mathrm{LE}^{\prime}}{\llcorner} \Gamma \Longleftrightarrow \text { there is a combinatorial } \mathrm{LE}^{\prime} \text {-proof } f: \mathcal{C}^{R \delta} \rightarrow \llbracket \Gamma \rrbracket
$$

Proof This follows from Theorems 7.1, 7.2, 8.6 and 9.2 .

Below is an example of a combinatorial LE'-proof. On the left the conclusion is shown as sequent, and on the right as weighted cograph.
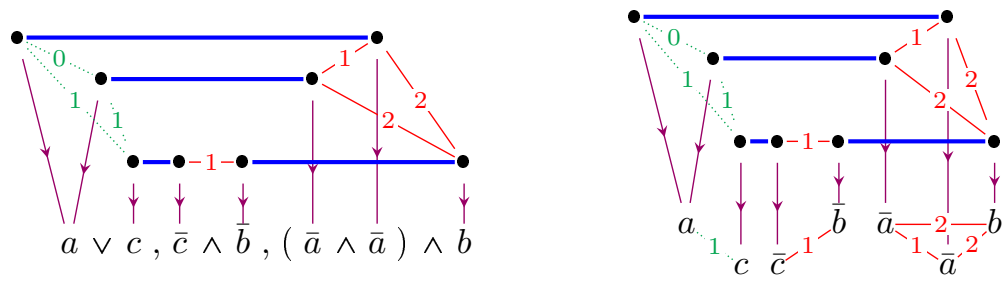

Theorem 9.5 Let $\Gamma$ be a sequent and $\mathcal{G}$ a graph together with a perfect matching and a weight function on its edges, and let $f$ be a map from $\mathcal{G}$ to $\llbracket \Gamma \rrbracket$. It can be decided in polynomial time in $\left|V_{\mathcal{G}}\right|+|\Gamma|$ whether $f: \mathcal{G} \rightarrow \llbracket \Gamma \rrbracket$ is a combinatorial $\mathrm{LE}^{\prime}$-proof. 
Proof All necessary properties (forbidden edges configurations for $\mathcal{G}$ being a weighted cograph, æ-connectedness and $æ$-acyclicity, and $f$ being a weighted relevant skew fibration can be checked in polynomial time.

\section{Conclusion}

In this paper we presented combinatorial proofs for entailment $\operatorname{logic} \mathbf{E}_{\mathfrak{\Im}}$, classical relevant logics $\mathbf{R}_{\Im}$ and classical relevant logic with mingle $\mathbf{R M}_{\Im}$. In some sense, combinatorial proof for entailment logic can be considered as a case study for logics with commutative but not associative connectives.

In fact, this paper can be seen as a small step in a larger research project showing that combinatorial proofs are a uniform, modular and bureaucraticfree way of representing proofs for a large class of logics. Apart from the logics studied in this paper, this goal has been achieved for multiplicative linear logic with and without mix in [15, for classical propositional logic in [1112 17, and for intuitionistic propositional logic in [?]. For first-order logic, modal logics, and larger fragments of linear logic, this is work in progress.

A necessary condition for a logic to have combinatorial proofs seems to be the ability to separate the multiplicative (linear) fragment from the additive (contraction+weakening) fragment. This can happen inside some form of deep inference proof system [6]10, and is realized in this paper in Theorems 3.6 and 7.2 .

A crucial condition that combinatorial proofs should obey, in order to be called combinatorial proofs for a chosen logic, is that all combinatorial properties needed for correctness of a given proof object can be checked in polynomial time with respect to its size. Then combinatorial proofs form a proof system (in the sense of Cook and Reckhow [7) for the chosen logic. The combinatorial proofs we give in this paper have this property.

Thanks to their combinatorial (or bureaucracy-free) nature, combinatorial proofs allow us to capture a less coarser notion of proof identity with respect to the one given by syntactic formalisms like sequent calculus and analytic tableaux. Following the work in [121,?] we put forward the following notion of proof identity:

Two proofs are the same iff they have the same combinatorial proof.

\section{References}

1. Acclavio, M., Straßburger, L.: From syntactic proofs to combinatorial proofs. In: International Joint Conference on Automated Reasoning. pp. 481-497. Springer (2018)

2. Anderson, A.R., Belnap, Jr., N.D.: Entailment, the Logic of Relvance and Necessity, Volume 1. Princeton University Press (1975)

3. Anderson, A.R., Belnap Jr, N.D., Dunn, J.M.: Entailment, Vol. II: The Logic of Relevance and Necessity, vol. 5009. Princeton University Press (2017)

4. Belnap, Jr., N.D.: Display logic. Journal of Philosophical Logic 11, 375-417 (1982) 
5. Belnap, Jr., N.D., Wallace, J.R.: A decision procedure for the system $e_{\bar{i}}$ of entailmengt with negation. Zeitschrift für Mathematische Logic und Grundlagen der Mathematik 11, 277-289 (1965)

6. Brünnler, K., Tiu, A.F.: A local system for classical logic. In: Nieuwenhuis, R., Voronkov, A. (eds.) LPAR 2001. LNAI, vol. 2250, pp. 347-361. Springer (2001)

7. Cook, S.A., Reckhow, R.A.: The relative efficiency of propositional proof systems. The Journal of Symbolic Logic 44(1), 36-50 (1979)

8. Duffin, R.: Topology of series-parallel networks. Journal of Mathematical Analysis and Applications 10(2), $303-318$ (1965)

9. Gentzen, G.: Untersuchungen über das logische Schließen. I. Mathematische Zeitschrift 39, 176-210 (1935)

10. Guglielmi, A., Straßburger, L.: Non-commutativity and MELL in the calculus of structures. In: Fribourg, L. (ed.) Computer Science Logic, CSL 2001. LNCS, vol. 2142, pp. 54-68. Springer-Verlag (2001)

11. Hughes, D.: Proofs Without Syntax. Annals of Mathematics 164(3), 1065-1076 (2006)

12. Hughes, D.: Towards Hilbert's $24^{\text {th }}$ problem: Combinatorial proof invariants: (preliminary version). Electr. Notes Theor. Comput. Sci. 165, 37-63 (2006)

13. McRobbie, M.A., Belnap, N.D.: Relevant analytic tableaux. Studia Logica 38(2), 187-200 (1979)

14. Möhring, R.H.: Computationally tractable classes of ordered sets. In: Rival, I. (ed.) Algorithms and Order, pp. 105-194. Kluwer Acad. Publ. (1989)

15. Retoré, C.: Handsome proof-nets: perfect matchings and cographs. Theoretical Computer Science 294(3), 473-488 (2003)

16. Straßburger, L.: A characterisation of medial as rewriting rule. In: Baader, F. (ed.) Term Rewriting and Applications, RTA'07. LNCS, vol. 4533, pp. 344-358. Springer (2007), http://www.lix.polytechnique.fr/ lutz/papers/CharMedial.pdf

17. Straßburger, L.: Combinatorial flows and their normalisation. In: Miller, D. (ed.) 2nd International Conference on Formal Structures for Computation and Deduction, FSCD 2017, September 3-9, 2017, Oxford, UK. LIPIcs, vol. 84, pp. 31:1-31:17. Schloss Dagstuhl - Leibniz-Zentrum fuer Informatik (2017) 